REVISTA INTERNACIONAL DE CIENCIAS DEL DEPORTE International Journal of Sport Science

Rev. int. cienc. deporte

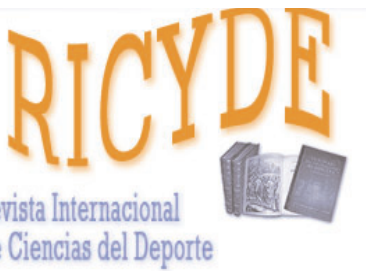

International Journal of Sport Science VOLUMEN X - AÑO X

Páginas:156-172 ISSN:1885-3137 No 36 - Abril - 2014

\title{
Las carreras populares en la Barcelona de la transición política (1975-1982) Popular races in Barcelona during the political transition (1975-1982)
}

\section{Sixte Abadia}

Universidad Ramón Llull. Barcelona

\section{Resumen}

Con la transición política española $(1975-1982)$ se inició un periodo de profundas transformaciones que también condicionó la realidad deportiva del país. En el caso de Barcelona, las carreras atléticas gozaron de un notable auge entre la población, erigiéndose en una manifestación deportiva característica de este periodo. Con su carácter popular, estas carreras se impregnaron de elementos constitutivos del momento, tales como la libertad o la democracia. Este artículo se centra en el análisis de las carreras populares en Barcelona como muestra de las transformaciones que vivió el fenómeno deportivo durante la transición política y, a su vez, como fenómeno a partir de la cual comprender algunos de los elementos constitutivos de la realidad social y política del momento. La metodología utilizada ha consistido en el análisis interpretativo de fuentes secundarias y hemerográficas. En este sentido, se pueden diferenciar dos periodos. El primero (1975-1978) permitió la aparición de las primeras carreras con un carácter participativo y festivo, mientras que durante el periodo 1979-1982 se vivió su auge y consolidación. Algunos de los elementos explicativos de esta expansión fueron la aparición de nuevas carreras, las connotaciones extradeportivas de éstas y la colaboración a nivel organizativo entre los diferentes agentes deportivos de la ciudad.

Palabras clave: carreras populares; deporte popular; democratización deportiva; transición política.

\begin{abstract}
With the Spanish political transition (1975-1982) a period of deep transformations began, which also affected the real sport situation of the country. In the case of Barcelona, athletic races had an important growth among the population, becoming a sporting event feature of this period. With its popular nature, these races were permeated by constituent elements of the time, such as the wish for freedom or democracy. This article is focused on the analysis of popular races in Barcelona as a demonstration of the transformations that the sporting event experienced during the political transition and also as an event from which we can understand some of the constituent elements of the social and political situation of that time. The methodology used has been the interpretive analysis of secondary sources and newspaper records. In this respect, it is possible to differentiate two periods: The first one (1975-1978) allowed for the appearance of the first races of a festive and participative nature, while in the period (1979-1982) it experienced growth and consolidation. Some of the reasons of this expansion were the appearance of new races as well as offfield elements and the organizational cooperation between the different sports agents of the city.
\end{abstract}

Key words: popular races; popular sport; democratization of sport; political transition. 
Abadia, S. (2014). Las carreras populares en la Barcelona de la transición política (1975-1982). RICYDE.

\section{Introducción}

L a transición democrática española (1975-1982) ha sido un objeto de estudio muy desarrollado en los últimos años desde las ópticas política, social, económica o cultural. No obstante, a excepción de las aportaciones de autores como Núria Puig (1993, 1995), Xavier Pujadas (1999) y Carles Santacana (2003) o Conrad Vilanou (1994), la realidad deportiva de este periodo ha sido poco estudiada.

El presente estudio busca corregir la escasez de estudios centrados en el análisis del fenómeno deportivo en un periodo de cambio como el de la transición política española. De este modo, se pretende mostrar como las carreras populares surgidas y desarrolladas en la ciudad de Barcelona entre 1975 y 1982 se erigieron en una importante expresión del cambio existente por aquel entonces y en un vehículo de democratización.

A menudo, la comprensión de un periodo histórico se aborda principalmente desde los prismas político y económico, ya sea desde un punto de vista de delimitación cronológica como de los principales hechos que explican los cambios más destacados del periodo estudiado. Sin negar la necesidad e importancia de estos análisis, es también cierta la necesidad de analizar las transformaciones sociales y ciudadanas para así tener una visión más precisa al respecto.

La transición política española, como un periodo de cambio de un régimen dictatorial como el franquista a otro de democrático que se puede enmarcar en la tercera ola de democratización (Huntington, 1994), permitió la movilización de múltiples sectores de la sociedad para conseguir una anhelada transformación política y la consecución de unas demandas largamente reivindicadas y silenciadas (Soto, 2005). En este sentido, la movilización social y la conflictividad laboral, la crisis económica y la violencia política fueron algunos de los principales condicionantes de este proceso (Soto, 2005). A su vez, desde un punto de vista social, la transición política permitió la consolidación de una sociedad de consumo y la creación del Estado del bienestar, coincidiendo con el cambio de mentalidad, de hábitos y de la valores de la ciudadanía, iniciado ya en España en las décadas de 1960 y de 1970 (Bernecker, 2009).

También a partir de la década de 1960, el fenómeno deportivo fue convirtiéndose en una actividad social de masas y en un hábito cotidiano (García, 2006), siendo la transición un período de inflexión (Abadia, 2011 a). Una de las principales características del deporte de la transición política fue la emergencia del deporte para todos, ejemplificado en la proliferación de carreras populares en el espacio urbano de las principales ciudades del país (Abadia, 2011 a). Fue también durante esta etapa cuando la dimensión competitiva del deporte fue cediendo protagonismo a favor de las dimensiones lúdica y formativa, algo que se reflejó en el citado deporte para todos y en la promoción deportiva en la tercera edad o en colectivos en riesgo de exclusión (Santacana, 2003).

Así pues, durante los años setenta y sobre todo los ochenta se dieron las condiciones idóneas para el inicio de un proceso de democratización deportiva (Puig, 1995), en el sentido de favorecer la expansión del fenómeno deportivo entre los diferentes estratos sociales y de normalizar "la situación deportiva en España, al formalizarse la enseñanza reglada de la educación física y aumentar el número de instalaciones y practicantes" (Moscoso, 2011, 113). Este proceso fue consecuencia de las acciones promocionales lideradas por entidades deportivas y movimientos sociales, y "sobre todo por parte de los Ayuntamientos democráticos a partir de la transición democrática" (García, 2006, 18). Esta voluntad de favorecer el acceso masivo al deporte por parte de la población ya se mostraba a comienzos de la transición en los discursos de los principales dirigentes del deporte español, siendo 
Abadia, S. (2014). Las carreras populares en la Barcelona de la transición política (1975-1982). RICYDE.

buen ejemplo de ello las declaraciones de Benito Castejón al afirmar que "la masificación en el deporte es otro de los propósitos de la Delegación Nacional, ya que pretende conseguir, una base sólida para alcanzar después una masa también de figuras notable" (Instalaciones deportivas en todos los pueblos españoles objetivo de la DND, 1976, 16). Este discurso modernizador y favorecedor de la extensión del deporte, fue consecuencia de la permeabilidad en los planteamientos deportivos del momento existentes a nivel europeo. La Carta Europea del Deporte para Todos (1975) fue ejemplo de ello (Moscoso, 2011), tal y como reconocía el mismo Castejón al considerar que "la educación física y la extensión del deporte para todos, son objetivos fundamentales de acuerdo con la carta del deporte del Consejo de Europa" (Declaración programática de la D.N.D., 1976, 3).

Durante este primer periodo post-dictatorial, el concepto de democratización también apareció ligado al deporte a partir de la necesidad de dotar de mayor transparencia y legitimidad democrática a las diferentes estructuras y organizaciones deportivas del país, siguiendo la estela del resto de esferas socio-políticas. Buen ejemplo de ello fueron la desaparición de la Delegación Nacional de Educación Física y Deportes -1 de abril de 1977y posterior creación del Consejo Superior de Deportes (CSD) -27 de agosto de 1977-, la convocatoria de elecciones a la presidencia de las federaciones deportivas o la reforma de los estatutos de numerosas entidades deportivas. Los medios de comunicación también recogieron esta inquietud, tal y como ilustra el titular "La democratización a encuesta" aparecido en Dicen... en octubre de 1976, y que recogía la opinión de los presidentes de las principales federaciones deportivas sobre las elecciones a sus cargos.

Este artículo tiene como finalidad ayudar a una mejor comprensión de lo acaecido durante esta época de cambio y evidenciar la importancia del fenómeno deportivo a partir del análisis de las carreras populares urbanas como expresión de modernidad, de libertad y de democracia. En definitiva, se pretende observar su contribución al desarrollo de unas condiciones favorables para la transformación democrática en la España del último cuarto del siglo XX.

\section{Método}

A nivel metodológico, esta investigación se ha articulado desde un punto de vista hermenéutico, principalmente a partir del análisis interpretativo de fuentes secundarias basadas en el estudio de la transición política y de la cuestión deportiva, así como de fuentes hemerográficas de temática deportiva como El Mundo Deportivo o Dicen.... El cuerpo del artículo se ha dividido en dos partes, la primera de las cuales permite una aproximación al comienzo de la transición política (1975-1978) y al inicio de cambio que experimentaron las carreras populares, para contextualizar una segunda mitad de la transición política (19791982) caracterizada por acontecer grandes modificaciones a nivel deportivo, entre ellas la consolidación definitiva de las carreras populares en la ciudad de Barcelona.

\section{Resultados}

\section{La proliferación de las carreras populares (1975-1978)}

Este primer periodo postdictatorial, iniciado con la muerte del dictador Franco, permitió la emergencia de nuevos planteamientos en materia deportiva, así como la progresiva incorporación del deporte entre las necesidades e intereses de la población. Estos cambios, evidenciados ya al final de la dictadura tal y como constataba el estudio realizado por el Instituto ICSA-Gallup (1975) a petición de la Delegación Nacional de Educación Física y Deportes, se acabarían de consolidar durante los años ochenta (García, 2001). Concretamente, durante el bienio 1977-1978 se dio la mayor transformación gubernamental 
Abadia, S. (2014). Las carreras populares en la Barcelona de la transición política (1975-1982). RICYDE.

en materia deportiva, siendo la desaparición de citada la Delegación Nacional (1 de abril de 1977) y la creación en agosto de 1977 del CSD uno de los hitos más remarcables, transformación que se vio acompañada de un cambio de discurso en relación a las prioridades deportivas.

Buena muestra de ello fue la especial atención hacia el deporte para todos (Vilanou, 1994) que se constató, por ejemplo, en la I Asamblea General del Deporte. En este encuentro, celebrado en diciembre 1977, se concluyó la necesidad de "instalaciones de 'deporte para todos' al que debe supeditarse el deporte espectáculo y el deporte de alto rendimiento" (Alfil, 1977, 20). Otro ejemplo fue la creación por parte del CSD de un Departamento de Promoción que pretendía "movilizar a grandes masas de población, de edades diversas, con poca o ninguna experiencia deportiva" (Marcos, 1981, 14). En este mismo sentido, la aprobación de la Constitución Española a finales de 1978, reconociendo el derecho de la ciudadanía al deporte mediante el artículo 43.3 (Martín, 1996, 107), evidenció la creciente sensibilidad hacia los déficits y reivindicaciones ciudadanas en materia deportiva.

En el caso de la ciudad de Barcelona y del deporte, la citada reestructuración gubernamental y el cambio en la concepción del modelo deportivo no acarreó a efectos prácticos demasiadas transformaciones. De este modo, el bienio 1977-78 fue importante no tanto por los avances y mejoras en la realidad deportiva de la ciudad, sino para el establecimiento de los fundamentos ideológicos que permitirían a partir de 1979 la materialización de políticas deportivas más orientadas a la democratización deportiva. Las carreras atléticas organizadas durante este periodo en el espacio público de la ciudad reflejaron esta dinámica, siendo más bien minoritarias y sin abarcar a amplios segmentos de población. El caso del I Cross C. N. Montjuïc - Gran Premi de la Generalitat (Barcelona despedirá el año corriendo, 1978, 24), organizado por el Club Natació Montjuïc y celebrado en el recinto de Can Borinot, sirve de ejemplo del carácter competitivo de algunos encuentros atléticos de la ciudad.

Ahora bien, este periodo también dio pié a las primeras muestras de una nueva expresión atlética como fueron las carreras populares. Fueron varias las asociaciones de vecinos de la ciudad que, en el marco de las fiestas mayores de barrio o de otras celebraciones, organizaron carreras urbanas con un destacado carácter participativo y festivo. Así, a través de la Asociación de Vecinos de Sant Andreu en el año 1977, y en el marco de la fiesta mayor del barrio, se celebró una carrera a pie y otra en bicicleta por un circuito por el barrio (Associació de Veïns de Sant Andreu, 1977, 10), o el año siguiente la Asociación de Vecinos del Distrito V organizó un festival de carreras populares, teniendo como centro neurálgico la Plaza Folch i Torres (Carreras populares en el distrito Quinto, 1978, 24). Un tercer ejemplo, en el marco de las Festes del Roser del barrio de Sarriá, fue el de la organización de una "carrera de footing" organizada por la asociación de vecinos, con una participación de 21 atletas (Associació de Veïns de Sarrià, 1978, 3).

Estos ejemplos ilustran uno de los ejes prioritarios de actuación vecinal como fue el de la recuperación del patrimonio y de las manifestaciones culturales del territorio, en buena medida reflejado en la voluntad de restauración y de potenciación de las fiestas populares de cada barrio. Fue en este marco donde el deporte con un carácter festivo, participativo y a menudo reivindicativo adquirió un papel cada vez más relevante, aunque durante este primer periodo de la transición aún quedó relegado a un segundo plano (Abadia, 2011 b). En la medida que sus demandas en relación a ejes prioritarios como la educación, la sanidad, el urbanismo, la limpieza de la ciudad, la defensa de la cultura catalana o de los derechos humanos se fueron resolviendo, creció la concienciación hacia la importancia de la práctica deportiva para la mejora de la calidad de vida de los barceloneses/as. Esto comportó la proliferación de iniciativas en relación al fenómeno deportivo. 
Abadia, S. (2014). Las carreras populares en la Barcelona de la transición política (1975-1982). RICYDE.

Otro síntoma de los cambios en eventos deportivos hasta entonces con un marcado carácter competitivo y en buena medida exclusivo, fue la carrera Jean Bouin. Ésta, organizada por el rotativo El Mundo Deportivo, en su edición de 1978 -la número cincuenta y cuatroincorporó el Primer Cross en Silla de Ruedas. De este modo se posibilitó que atletas con alguna deficiencia motriz disputaran una prueba de seis-cientos metros de distancia (40 minusválidos correrán en silla de ruedas, 1978, 27). Esta carrera se realizó acompañada de "incontables aplausos de todos los espectadores" y de una emoción que "ha invadido un poco a todos, al ver a los 41 participantes llegando uno tras otro, animados por compañeros y amigos" (Muntaner, 1978, 35). Así fue como esta tradicional carrera barcelonesa inició su transformación definitiva de acuerdo con el nuevo rumbo más cercano al deporte para todos, que poco a poco cuajó en las sociedades española y barcelonesa.

Durante estos años, quizás el evento que tuvo un seguimiento más masivo fue el organizado por la Sociedad Naturista y Vegetariana de Barcelona. Con claro perfil reivindicativo, en diciembre de 1977 esta organización lideró "una manifestación en bicicleta a través de prácticamente todo el 'núcleo' de la ciudad, con parada momentáneamente y entrega en la Generalitat de un escrito" que exigía "carriles para bicis en calles y plazas; transportes dignos y suficientes; poner límite a las edificaciones masivas; aumento de zonas verdes y ordenación racional y popular del territorio" (Dalmases, 1977, 22). El éxito fue tal que en su primera edición congregó a más de 40.000 ciudadanos que anhelaban "convertir la jungla de Barcelona en un espacio más o menos habitable donde sencillamente el vivir no resulte un auténtico martirio" (Dalmases, 1977, 22). Un año más tarde, la segunda Diada Naturista, con el eslogan "Lluitem per una nova vida" [Luchemos por una nueva vida], también fue un gran éxito y se hizo extensible a otras ciudades de Catalunya.

De este modo, durante el bienio 1977-1978, de manera incipiente y como proemio de los que sucedería a partir de 1979, se empezaron a observar iniciativas deportivas atléticas con un marcado carácter popular, festivo o reivindicativo. Su éxito, en el marco de un contexto de recuperación de las libertades individuales y colectivas tras la dictadura (Pujadas y Santacana, 1999), se reflejó en la gran capacidad de convocatoria y de participación que progresivamente consiguieron. Hasta entonces las posibilidades deportivas en el espacio público de la ciudad quedaban reducidas a manifestaciones deportivas de carácter competitivo y excepcional, como la Volta a Catalunya, las 24 Horas de Montjuïc, la Travesía al Puerto de Barcelona o la Pujada al Castell de Montjuïc.

\section{El auge y consolidación de las carreras populares en Barcelona (1979-1982)}

Tras los cambios organizativos y de índole estructural en el deporte español, el año 1979 arrancó con unas elecciones municipales -3 de abril de 1979- que consolidaron definitivamente el marco organizativo del deporte español (Puig, 1993, 96), y que permitieron la descentralización de la acción gubernamental hacia una mayor asignación de competencias y responsabilidad municipales. Al igual que otras importantes ciudades españolas, en el caso de la ciudad de Barcelona la victoria fue del Partit dels Socialistes de Catalunya (PSC-PSOE). El nuevo consistorio liderado por Narcís Serra dejó entrever la poca atención prestada por sus antecesores hacia la cuestión deportiva. El mismo Albert Pons Valón, concejal de deportes de la ciudad a partir de 1979, admitía que hasta entonces el Ayuntamiento sólo se había limitado a mantener "en funcionamiento de las instalaciones existentes, conceder alguna que otra subvención y patrocinar algún trofeo" sin "asumir la responsabilidad de cara a una auténtica promoción del deporte a escala ciudadana" (Astruells, 1979, 25). 
Abadia, S. (2014). Las carreras populares en la Barcelona de la transición política (1975-1982). RICYDE.

Al igual que en buena parte del territorio español, tras estas elecciones se desplegaron una serie de medidas que poco a poco configuraron una verdadera política deportiva municipal. Durante esta primera legislatura los dos principales objetivos en materia deportiva fueron "situar la actividad física y el deporte al alcance de la mayor parte de los ciudadanos" y "convertir Barcelona en el marco más idóneo para que sean acogidas grandes manifestaciones deportivas, generando riqueza, interés y espectáculo" (Ajuntament de Barcelona, 1983, 173). Estos dos ejes de actuación se materializaron de manera multidimensional en la realidad deportiva de la ciudad, siendo a su vez prioritaria la consecución de una mayor "rentabilidad de las instalaciones deportivas, así como la promoción preferente de la práctica deportiva" y "el fomento del deporte escolar" (Gaseta Municipal de Barcelona, 1979, 722). En paralelo a la continuidad en la mejora de una red de instalaciones deportivas ajustada a las necesidades de la población, la tarea municipal se centró en la organización de eventos deportivos de carácter popular y en la recuperación de espacios al aire libre para la práctica deportiva. Para ello se asignaron partidas presupuestarias centradas en la promoción de actividades deportivas, que en el caso del bienio 1979-1980 ascendieron a los 9.850.000 de pesetas, a las que se podían añadir 11.655.000 de pesetas más en concepto de subvenciones a entidades promotoras. Desde un punto de vista crítico, esta mayor implicación municipal hacia cuestiones deportivas podía interpretarse como una manera de sustituir el creciente déficit de espacios públicos a disposición de toda la ciudadanía (Puig, 1981) e incluso generar una pugna "entre el protagonismo del Ayuntamiento y el del movimiento asociativo" (Segura, 1981, 36).

Esta mayor implicación municipal hacia la gestión del deporte también se evidenció en la creciente colaboración con el resto de agentes promotores del deporte, ya fuesen de índole meramente deportiva -federaciones, clubes, asociaciones deportivas...- o no -empresas de servicios, asociaciones de vecinos...-. De este modo, estos años post-dictatoriales también permitieron el asentamiento de las bases de un modelo de relación cooperativo entre los poderes públicos y la sociedad civil (Puig y Heinemann, 1995).

Una de las manifestaciones en las que se mostró este nuevo rumbo de las políticas deportivas municipales así como la voluntad de colaborar -a nivel logístico, económico o mediante asesoramiento- con el resto de agentes del sector deportivo, fue el de las carreras populares (Abadia, 2011 a). Éstas, como clara expresión del deporte para todos, tuvieron en la segunda mitad de la transición democrática un periodo en el cual se dieron unas condiciones favorables para su expansión por todo el país.

En el caso de Barcelona, tras unos primeros años de transición democrática en los cuales habían aparecido ya las primeras carreras con un marcado carácter popular y participativo, a partir de 1979 se vivió una etapa de auge a partir de su proliferación por toda la ciudad y éxito de convocatoria.

A continuación se destacan los principales ejes explicativos de estas carreras en el marco de la realidad social, política y deportiva de la Barcelona de los años 1979-1982.

Una nueva manera de entender el deporte: el carácter participativo y urbano de las carreras populares

A partir de 1979 se dieron las condiciones necesarias para que las carreras populares emergiesen definitivamente e iniciasen su rápida consolidación como práctica deportiva en el espacio público de la ciudad. La mayor madurez democrática transcurridos ya unos años desde la muerte del dictador, la reestructuración gubernamental y la consiguiente celebración de las elecciones municipales de ese mismo 1979, conjuntamente con una ciudadanía cada vez más cercana a la cuestión deportiva y con ansias de libertad y de participación en 
Abadia, S. (2014). Las carreras populares en la Barcelona de la transición política (1975-1982). RICYDE.

cualquier celebración popular, permitieron el establecimiento de unas condiciones favorables para esta nueva manera de practicar deporte.

La primera muestra de este tipo de carreras fue iniciativa de El Corte Inglés, una conocida cadena de establecimientos comerciales que organizó la $1^{\text {a }}$ carrera popular "Ciutat de Barcelona" como "acto ciudadano destinado a la juventud", según afirmaba Jorge Pinto, relaciones públicas de estos establecimientos (Barcelona se pone a correr con la I carrera popular, 1979, 31). Su marcado carácter participativo se reflejaba en la misma difusión de la carrera con una inscripción "abierta y sin límite de edad", algo que incluso sorprendió a los organizadores al observar "la presencia de familias enteras" o "de un inscrito de 5 años y otro de 84 años", que permitieron llegar a la cifra de 17.184 participantes en su primera edición (Barcelona se pone a correr con la I carrera popular, 1979, 31).

En el marco de la Fiesta Mayor de la ciudad, las Fiestas de la Mercè, se pudo comprobar que el éxito participativo de la carrera "Ciutat de Barcelona" no era casual. El Ayuntamiento entrante promovió la I Cursa Atlètica de la Mercè, contando la colaboración de varias organizaciones. En este caso fueron unos 15.000 participantes, reflejando también el carácter popular de esta carrera al aglutinar "a recordman nacionales, a hombres de más de 70 años y a niñitos de 3, a presidentes de secciones atléticas..." (25.000 Barceloneses, en la "Cursa de la Mercè”, 1979, 27).

La tercera muestra de este auge atlético, ya a finales de ese mismo 1979, fue el correspondiente a la Jean Bouin que, tras haber incluido algunas modificaciones en sus anteriores ediciones, celebró por primera vez una carrera "open" para no federados, sumándose "a la corriente a favor de la carrera a pie, existente en nuestros días, no en plan de competición sino más bien de práctica atlética" (Jean Bouin: La Carrera Popular, novedad de este año, 1979, 30).

El éxito y el carácter que adoptaron estas tres carreras fue una buena muestra que el fenómeno deportivo dejaba de ser exclusivamente competitivo para extenderse a todos los sectores sociales de la ciudad. Su continuidad siguió caracterizándose por este carácter participativo, tanto por el número de corredores como por su heterogeneidad. En los primeros años de la década de los ochenta la prensa escrita habitualmente destacaba ambas cuestiones. Por ejemplo, la Cursa Popular "Ciutat de Barcelona" era considerada "la primera manifestación de participación catalana" al contar en su segunda edición con 29.953 corredores inscritos (En marcha, la II carrera popular "Ciutat de Barcelona", 1980, 38), cifra que en 1982 se elevó hasta los 33.856 atletas. En esa edición, de los 31.772 participantes no federados destacaban la presencia de "17 pilotos, 136 médicos, 20 veterinarios, 49 farmacéuticos, 32 matemáticos, 141 economistas, 59 abogados, 421 maestros, 19 sacerdotes y 2 religiosas, 71 músicos, 196 funcionarios del Estado, 200 miembros de las fuerzas armadas, 1248 vendedores, 3300 administrativos, 633 agricultores, 123 empleados del servicio doméstico, 20201 estudiantes" (Cánovas venció en la "Cursa" de "El Corte Inglés", 1982, 36).

Las otras dos carreras, la Cursa Atlètica de la Mercè y la Jean Bouin, se caracterizaron también por su gran y heterogénea participación. En este sentido, la edición de 1980 de Cursa Atlètica de la Mercè contó con "una crecida participación de mujeres de toda edad, como también de atletas minusválidos, y aun de familias completas, con los hijos en cochecitos de ruedas" (Segona Cursa de la Mercè. Toda Barcelona se puso a correr, 1980, 30).

El carácter participativo se reflejaba en la voluntad y sensibilidad hacia el aumento del número de mujeres. Su presencia era aún minoritaria, como se constató en la Cursa de El Corte Inglés -como popularmente se conocía la Cursa Popular "Ciutat de Barcelona"- de 
Abadia, S. (2014). Las carreras populares en la Barcelona de la transición política (1975-1982). RICYDE.

1981, que contó con algo más de 6.000 mujeres, de un total de 24.953 participantes (C. M., 1981, 41). También desde el Ayuntamiento de Barcelona y la Cursa Atlètica de la Mercè se buscaba estimular la participación femenina premiando a las nueve primeras clasificadas en las distintas categorías.

El éxito participativo y la dimensión alcanzada por estas tres carreras no debe concebirse como un fenómeno aislado, sino que respondió a una tendencia de ocupación del espacio público mediante la proliferación de eventos deportivos en la ciudad con un acentuado tono festivo, ya fuesen propuestas de carácter más multitudinario y de gran envergadura como menos masivas. Todas ellas permitieron una creciente dinamización del tejido social barcelonés hacia la participación deportiva, al tiempo que la ciudad se fue dotando de nuevos espacios deportivos (Pujadas y Santacana, 1999).

Tabla 1: Número aproximado de participantes en las cuatro principales carreras atléticas de Barcelona durante la transición política

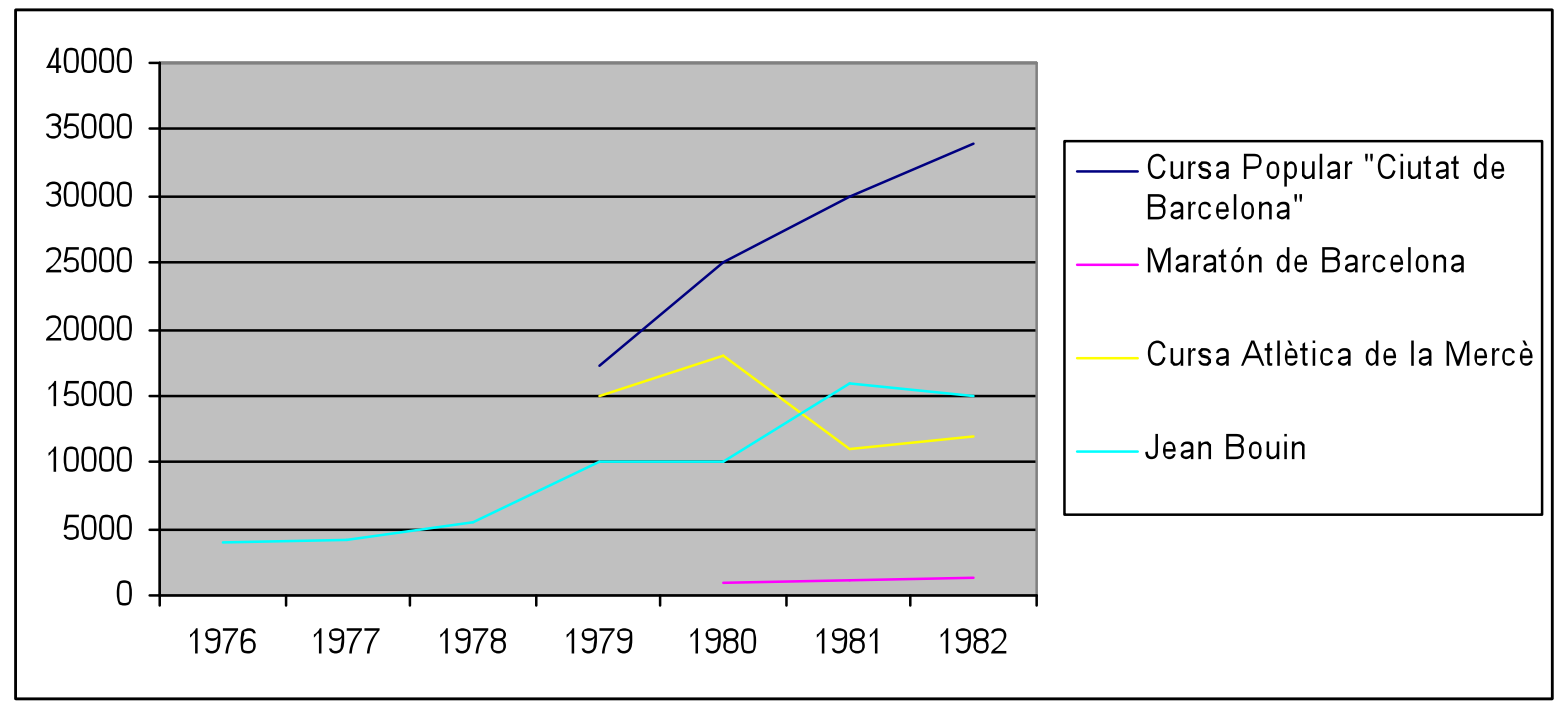

Desde instancias políticas, estas manifestaciones deportivas eran algo más que carreras populares. Por ejemplo, Josep Lluís Vilaseca, en calidad de director general del deporte de la Generalitat de Catalunya, consideraba que la Jean Bouin era "una de la grandes celebraciones con que cuenta Barcelona y Catalunya entera", "siendo una de las competiciones que con mayor ahínco contribuyeron a la difusión del deporte" (Vilaseca: "Un encuentro con la tradición”, 1980, 48). También Enric Truñó, como máximo responsable del Área de Juventud y Deportes del Ayuntamiento a partir de 1981, destacaba la importancia de la Cursa Atlética de la Mercè "para que Barcelona siga demostrando amor hacia el deporte" y "para que el ciudadano participe activamente en el desarrollo del espíritu deportivo que tiene que marcar la vida de la ciudad" (Presentación oficial de la "IV Cursa Atlética de la Mercè", 1982, 31).

En este sentido, ya durante la segunda mitad de la transición democrática existía la sensación que correr por la ciudad había dejado de ser algo residual producto del boom atlético existente en España, un "fenómeno que ha llegado desde las Américas y que se llama 'footing"' (E.M., 1980, 25). Ciertamente, la influencia americana del jogging fue importante en el desarrollo de las carreras populares. En el caso de Barcelona, quizás las muestras más palpables de ello fueron el Maratón de Barcelona y la figura de Ramón Oliu. Éste, llegó a Barcelona procedente de los Estados Unidos y como buen aficionado al jogging fundó en 1977 la Comisión Marathon Catalunya y fue el director del Maratón en sus primeras ediciones. Además, fue alguien muy activo en el fomento de las carreras populares. Ejemplo de ello fue la organización, conjuntamente con Pere Pujol y Jaume Raventós, de diferentes 
Abadia, S. (2014). Las carreras populares en la Barcelona de la transición política (1975-1982). RICYDE.

simposiums sobre maratón y cuyas principales aportaciones fueron recogidas en la publicación Del "jogging” a la marathon (1981). Esta influencia se detecta también en la aparición en España de ediciones de algunas publicaciones centradas en este fenómeno, como Correr para vivir mejor de Bob Glover y Jack Shepherd (1979), Jogging de James Hewitt (1980) o La práctica del jogging de Toby Hempel (1981).

El éxito participativo de estas carreras era una evidencia más que en "el año que finaliza se puede decir que no ha habido barriada ni pueblo que haya dejado de organizar su carrera popular, donde se han mezclado en muchas ocasiones los atletas federados [...] con los que forman en la ingente legión de practicantes del 'jogging' y del 'footing'” (Jean Bouin: La Carrera Popular, novedad de este año, 1979, 30). Este apogeo coincidía con la construcción de seis circuitos naturales -conocidos también como circuitos de footing- en distintos parques y zonas naturales de la ciudad, en el marco de la Campaña Nacional Deporte para Todos promovida por el Consejo Superior de Deportes que tuvo lugar en las principales ciudades de España (Marcos, 1981).

Y es que durante estos años la diversidad de eventos deportivos celebrados en el espacio público fue amplia. Buen ejemplo de ello fueron la Festa de la Bicicleta, organizada por el Ayuntamiento de Barcelona desde 1980, o la I Maratón Popular sobre Patines. Esta última, organizada en septiembre de 1980 por El Periódico y Patín Sport, contó con unos 3.200 patinadores entre los 2 y los 70 años (Messeguer se impuso en la "Marathon", 1980, 36). La Festa de la Bicicleta en sus primeras ediciones consiguió también una importante participación, tal y como reflejan los 4.000 ciclistas que el año 1981 "se dieron cita en el Parque de la Ciutadela para hacer esos 20 kilómetros de recorrido [...] en un día espléndido para el ejercicio" (Carbonell, 1981, 47). Esta nueva tendencia de práctica deportiva se caracterizaba también por realizarse al margen de estructuras asociativas y federativas, en buena medida por tratarse "de una actividad en solitario o, como máximo, en familia" (Puig, 1981, 59).

Aunque de carácter menos multitudinario, otra muestra del nuevo rumbo que estaba adquiriendo el deporte se observó en la proliferación de carreras organizadas por entidades deportivas y por asociaciones vecinales o culturales, a menudo en el marco de conmemoraciones o festividades. Estas carreras desarrollaron una notable función de acercamiento de la práctica deportiva a la población y en su articulación alrededor de manifestaciones festivas, identitarias, reivindicativas o meramente deportivas.

Su citado carácter participativo derivó en una necesidad de adecuar el lenguaje utilizado para calificar las carreras atléticas. La emergencia de la "Ciutat de Barcelona" o la Cursa Atlètica de la Mercè obligó a reflexionar sobre denominación de este tipo de carreras y de sus participantes en comparación con las carreras clásicamente competitivas protagonizadas por atletas federados.

Así, si las carreras "Ciutat de Barcelona" y Atlètica de la Mercè podían calificarse de meramente populares, ya que "el fin primordial de la carrera es el de participación" (F. C., 1982, 26), la Jean Bouin a partir de su edición de 1979 conjugaba "algo tan importante como es la participación y la competición" (La gran fiesta atlética, 1980, 38), tras una edición, la de 1979, en la cual esta carrera se sumó "a la corriente a favor de la carrera a pie, existente en nuestros días, no en plan de competición sino más bien de práctica atlética” (Jean Bouin: La Carrera Popular, novedad de este año, 1979, 30). Esta necesidad de conceptualización llegaba al extremo de intentar diferenciar los participantes de la edición de 1982 de la Jean Bouin entre "unos dos mil aficionados a estar en forma" y "otros pocos al atletismo" (La gran fiesta atlética, 1980, 38). 
Abadia, S. (2014). Las carreras populares en la Barcelona de la transición política (1975-1982). RICYDE.

Desde el punto de vista de autores como Casasa y Durà (1984), la emergencia y el éxito de estas carreras no podía concebirse como un fenómeno aislado y susceptible de ser criticado por su carácter puntual y engañoso. En contra de las carreras populares, estos mismos autores argumentaban el hecho de ser un "gran espectáculo colectivo, que quizás saldrá en los medios de comunicación, que será utilizado por los organizadores como mérito en el campo del deporte" (Casasa y Durà, 1984, 128) y la inexistencia de una preparación deportiva adecuada en la mayoría de participantes.

El auge adquirido por estas carreras populares formó parte de un fenómeno más amplio enmarcado en el inicio del primer boom atlético y en una ocupación del espacio público de la ciudad para una práctica deportiva que se iba consolidando como hábito deportivo y como forma de expresión ciudadana.

\section{Construyendo un futuro y una ciudad mejor: libertad, democracia y reivindicación}

Desde Comisión Marathon Catalunya se destacaba el carácter de una carrera en la cual "no quedemos manifestaciones, reivindicaciones o payasadas" (Muntaner, 1981, 14). Este fragmento es ilustrativo del carácter de esta prueba y a su vez de las diferencias existentes entre las carreras más multitudinarias de la ciudad.

Algunas de ellas, como la "Ciutat de Barcelona" o la Cursa de la Mercè, se caracterizaron por su carácter participativo y multitudinario, así como por la reivindicación espontánea de algunos de sus participantes. Por el contrario, en la Jean Bouin y el Maratón de Barcelona no proliferaron muestras de protesta, en buena medida por su mayor carácter competitivo.

Ejemplo de ello se dio en la I edición de la "Ciutat de Barcelona" cuando dos participantes desplegaron ya cerca de meta una pancarta con el lema "Magisterio en lucha". A menudo el carácter mediático e interés generado por estos eventos, en un contexto de reivindicación como fue el de la transición democrática, fue aprovechado para dar a conocer una problemática concreta o explicitar algún tipo de mensaje.

Cabe recordar que durante la transición democrática la sociedad civil ejerció un papel destacado en el sentido de condicionar un proceso de naturaleza política mediante su movilización y el voto (Soto, 2005). Las carreras populares, lejos de quedar exento de las características del periodo, fue un medio a través del cual colectivos muy diversos hicieron sentir su malestar y reivindicación hacia determinadas cuestiones.

En paralelo a las protestas más bien espontáneas y de poca repercusión en el marco de carreras como la Ciutat de Barcelona, fueron las asociaciones de vecinos las que ejemplificaron de manera más clara el carácter reivindicativo con el cual se acercaron al fenómeno deportivo, y en concreto a las carreras populares.

Estas asociaciones de vecinos, arraigadas en la ciudad definitivamente durante la transición democrática a partir de un sentimiento común de lucha y de defensa de unas mejores condiciones de vida (Huertas y Andreu, 1996), se acercaron gradualmente a la cuestión deportiva en la medida que se obtuvieron otras demandas sociales. Uno de estos casos lo encontramos en la Associació de Veïns del Parc que a mediados de 1980 organizó "una Mini Marathon Familiar, desde los 8 años, como primer eslabón para más adelante fomentar, al amparo de la Asociación, un equipo de footing" (Associació de Veïns del Parc, 1980, 10).

Ahora bien, más allá de la voluntad de favorecer la práctica deportiva vecinal, a menudo la organización de carreras populares fue el pretexto para otras reivindicaciones, como sucedió en varias ediciones de la Cursa Popular al Poble Sec per la Salut i el Medi Ambient celebradas a partir de 1980. Otro ejemplo en este mismo sentido fue el "Footing per la salut" 
Abadia, S. (2014). Las carreras populares en la Barcelona de la transición política (1975-1982). RICYDE.

que, en el marco de la Semana de la Salud, organizó la Federació d'Associacions de Veïns de Barcelona (FAVB) a mediados de 1980 (Fotut pel footing, 1980, 9), y que finalizó con la lectura de un manifiesto que incidía en la salud escolar o laboral y criticaba el proyecto de reforma sanitaria que aprobó Unión de Centro Democrático (UCD).

Este tipo de reivindicaciones no fue exclusivo de las asociaciones de vecinos. También otras organizaciones se acercaron a las carreras populares con un afán de reivindicación. Este fue el caso del Ateneo Sempre Avant, que en septiembre de 1979 organizó el I Cross Popular coincidiendo con la Fiesta Mayor del barrio de Sants con la voluntad de reivindicar instalaciones deportivas municipales para "disponer de un lugar propio para hacer gimnasia". Desde esta organización se afirmaba que "seguiremos luchando, utilizando como siempre nuestras armas, que son la cultura y la imaginación, para que este deseo se convierta en realidad bien pronto" (Ateneu Enciclopèdic "Sempre Avant", 1979).

Otro elemento a considerar es la contribución del deporte y particularmente de las carreras populares a la configuración de una mayor identidad de barrio y de ciudad. Así lo afirmaba a finales de 1982 Pasqual Maragall, el sucesor de Narcís Serra al frente de la alcaldía de Barcelona, al declarar en relación a la carrera Jean Bouin que "pruebas como esta 'fan ciutat'. La 'Jean Bouin' es algo entrañable para todos los barceloneses. Es una prueba que funciona, la respuesta por parte de los participantes se da de un modo total y absoluto" (Duran, 1982, 39).

Desde esta clave identitaria, las asociaciones de vecinos tuvieron también un papel muy destacado en la creación de una identidad colectiva alrededor de las aspiraciones y necesidades ciudadanas. Su labor de estimulación del tejido social de cada barrio de Barcelona fue importante en esta configuración identitaria, también para la población que a partir de la década de los años cincuenta llegó a la ciudad, y para los cuales su participación en las asociaciones de vecinos como espacio social de articulación de identidades fue básica para su integración (Nash, 2002).

En este sentido, en la medida que el deporte $-\mathrm{y}$ entre sus manifestaciones las carreras populares- fue incorporándose en la lógica de actuación vecinal, éste se convirtió también en un elemento favorecedor de la identidad vecinal. Sin ir más lejos, la progresiva aparición de vocalías de deporte como espacio de articulación vecinal, fundamentaban la existencia de vínculos de identidad y de pertinencia vecinal alrededor de la práctica deportiva.

Así pues, las carreras populares de este periodo, no tan sólo fueron una expresión de la evolución de la cuestión deportiva, sino también de la realidad de un periodo en el cual la ciudadanía se caracterizó por la movilización. Ésta tuvo en este tipo de manifestaciones un vehículo de reivindicación democrática y de construcción identitaria, a menudo, con un claro carácter festivo.

\section{De ensayos y apoyos: unos años de aprendizaje organizativo y de respaldo político}

La emergencia de las carreras populares también se caracterizó por convertirse en un periodo de iniciación a la organización de este tipo de eventos. Durante la transición se tuvo que hacer frente a la inexperiencia organizadora, algo que derivó en la aparición de algunos errores y en la constante introducción de modificaciones organizativas que paulatinamente permitieron el buen desarrollo de estas manifestaciones deportivas.

Esto es lo que sucedió en la Cursa de la Mercè de 1980, de la cual en una crónica de El Mundo Deportivo se afirmaba que "la organización de este año dejó mucho que desear" al existir problemas en la salida de una de las carreras programadas en la cual "los atletas 
Abadia, S. (2014). Las carreras populares en la Barcelona de la transición política (1975-1982). RICYDE.

partieron siete minutos antes de hora, sin que nadie pudiese dar la salida oficial" (Segona Cursa de la Mercè. Toda Barcelona se puso a correr, 1980, 30).

En ocasiones la prensa escrita iba más allá y no dudaba en afirmar en relación con la edición de 1981 de la Jean Bouin que "la sensación que nos ha dado, particularmente, es que los organizadores se ven desbordados" al constatar que "participantes, organización, jueces y en general todos los involucrados, terminan enfadados unos con otros" y que "un año por una cosa, otro por otra, está visto que la Jean Bouin cada temporada debe acabar con problemas" (Muntaner, 1980, 32).

Esta carrera no era la única que presentó problemas en sus primeras ediciones. En este sentido, una de las preocupaciones de la $3^{\text {a }}$ edición de la "Ciutat de Barcelona" era contener a los corredores en la salida para que ésta se efectuara en la hora prevista. Según Jordi Pintó, uno de los responsables de la prueba, "hemos tenido muchas reuniones sobre el tema, y es cosa muy difícil contener en la salida a tantísima gente [...], por lo que apelamos a todos los participantes para que se autorresponsabilicen" (F.C., 1981, 31).

$\mathrm{Y}$ es que las primeras ediciones de estas carreras estaban sometidas a continuos cambios. Por ello, en las ediciones de 1981 y de 1982 de la Cursa de la Mercè se optó por efectuar "las salidas desde un mismo lugar, pero con horarios distintos, a fin que los atletas no iniciados tuviesen que verse siempre postergados ante la presencia de los atletas federados", algo considerado como "un nuevo ensayo" (La IV "Cursa" de la Merced, record de participación, 1982, 24).

Esta inexperiencia organizativa, derivó también en la necesidad de capacitar a los organizadores y de generar conocimiento sobre la temática deportiva. En esta óptica se enmarca la aparición de cursos y de publicaciones centradas en la organización de eventos deportivos.

Cabe destacar los cursos organizados en Barcelona por el CSD entre diciembre de 1978 y enero de 1979 a través de su Delegación Provincial de Barcelona, centrados en el deporte escolar y en el deporte para todos, como el "Curso de dirigentes deportivos escolares", el "Curso de actualización de educación física escolar" o el "Curso monográfico 'Deporte para Todos"” (Cursos del CSD en Barcelona, 1978, 30). En este mismo marco y organizados por el Ayuntamiento de Barcelona, se diseñaron otros cursos "destinados a elevar el nivel de los que trabajan en la promoción y la enseñanza de las actividades física y deportivas", como el "Curso de deporte recreativo en el municipio", las "Primeras jornadas sobre deporte escolar" y las "Actividades física para la tercera edad".

De este modo, las diferentes administraciones se mostraron sensibles a las necesidades de formación deportiva. Este fue el caso también de la Diputación de Barcelona, que entre sus ámbitos de actuación y finalidades tenía la organización de cursos y seminarios de asesoramiento técnico y deportivo para los municipios de la provincia.

A nivel estatal también se publicaron algunos textos centrados en las pautas organizativas a tener en cuenta para la organización de carreras populares, al constatarse que "la organización de una actividad popular, con participación masiva, requiere no sólo de cierta dosis de experiencia, sino una gran imaginación y conocimiento minucioso de lo que se quiere conseguir" (Marcos, 1981, 18). De este modo, se pretendía responder a las necesidades formativas y de orientación existentes. Algunas de la consignas facilitadas del tipo "los mejores días suelen ser los domingos y festivos, o los coincidentes con las fiestas de la localidad respectiva", "la mejor manera de conseguir el éxito total es la de crear un Comité de Organización”, "uno de los aspectos más importantes de las actividades populares es, sin 
Abadia, S. (2014). Las carreras populares en la Barcelona de la transición política (1975-1982). RICYDE.

duda alguna, el reconocimiento previo y la asistencia médica durante la prueba" o "los participantes no rehúsan jamás colaborar económicamente con los organizadores, mediante la aportación de alguna cantidad, que no suele ser superior a las 150 pesetas" (Marcos, 1981, 18-20), se presentaban como necesarias en ese momento.

Así pues, la emergencia y rápida consolidación de las carreras populares en la ciudad de Barcelona supuso un reto organizativo condicionado por el éxito de las mismas, algo que también derivó en la necesidad de establecer pautas organizativas que permitiesen una mejor gestión de estos eventos deportivos. En este sentido, fue básica la colaboración entre el tejido asociativo y las diferentes administraciones.

\section{Conclusiones}

Podemos destacar tres ideas principales sobre la realidad de las carreras populares de Barcelona durante la transición política española (1975-1982).

La primera de ellas es que la evolución de este tipo de manifestaciones deportivas se caracterizó por algunos de los mismos elementos configuradores del periodo de cambio estudiado. Desde este punto de vista, la transición política conllevó la adopción y arraigo de nuevos hábitos y valores en un marco de creación del Estado del bienestar, así como una creciente conflictividad social, en buena medida como consecuencia de la crisis económica existente y de las demandas sociales tras décadas de dictadura. A nivel deportivo, la reestructuración iniciada entonces permitió la emergencia de una nueva concepción de deporte y su consiguiente democratización o expansión entre la ciudadanía (García, 2006; Moscoso, 2011; Puig, 1995). Para ello el papel de las diferentes administraciones fue clave, inicialmente en la adopción de discursos próximos a una concepción de deporte para todos con una clara influencia de la Carta Europea del Deporte para Todos (1975)- y en el progresivo reconocimiento del deporte como necesidad y derecho ciudadano -Constitución Española (1978). Así pues, cabe concebir la transición política como un periodo esencial de cara a la evolución deportiva del país, a partir de la progresiva transformación de la ciudadanía y del cambio de rumbo político.

Un segundo aspecto a destacar, derivado de esta reflexión inicial, es la importancia que tuvieron las carreras populares como ejemplo de democratización deportiva y como reflejo de la realidad social de la transición. Más allá de ejemplificar el progresivo acercamiento al deporte de la población barcelonesa, su aparición y éxito en ocasiones fue un reflejo de las ansias de libertad, de democracia y de reivindicación de la población. En este sentido, la ocupación del espacio público de la ciudad para todo tipo de manifestaciones deportivas de carácter popular -destacando especialmente las carreras atléticas- permitió ganar un nuevo emplazamiento deportivo, hasta entonces reservado a eventos de marcada índole competitiva, y sirvió de ejemplo del nuevo contexto de libertad y de democracia iniciado.

Una tercera y última reflexión es la correspondiente a la propia evolución de las carreras populares, observándose dos momentos claramente diferenciados. Un primer periodo (19751978) en el cual el espacio público de la ciudad acogió las primeras carreras participativas y festivas, observándose también la transformación de carreras consolidadas como la Jean Bouin hacia modelos más participativos y acordes con los nuevos hábitos ciudadanos. Es también durante estos primeros años post-dictatoriales cuando el movimiento vecinal de la ciudad organiza las primeras carreras con voluntad reivindicativa y festiva, a manudo en un marco de recuperación de las fiestas mayores. A partir de las elecciones municipales del 3 de abril de 1979, en Barcelona se configura una política deportiva municipal orientada hacia la democratización deportiva y a los grandes eventos, basándose en un modelo de relación 
Abadia, S. (2014). Las carreras populares en la Barcelona de la transición política (1975-1982). RICYDE.

cooperativo entre la administración y el resto de agentes deportivos. Con esta nueva orientación se inició una nueva etapa, caracterizada por la notable expansión de las carreras atléticas. Un eje explicativo de su desarrollo fue la gran aceptación que tuvieron carreras como la "Ciutat de Barcelona" o la Cursa Atlètica de la Mercè, ambas organizadas por primera vez en 1979 y con una vocación plenamente festiva y plural. Su éxito sirvió para evidenciar una evolución hacia modelos deportivos que dejaban de ser eminentemente competitivos. A estas carreras más multitudinarias se sumaron las organizadas por todo tipo de asociaciones deportivas, culturales o vecinales en el marco de cada barrio de la ciudad. Sin ser tan multitudinarias, éstas fueron importantes para el acercamiento de la práctica deportiva cotidiana a la población y como vehículo identitario y de reivindicación. De este modo, se puede afirmar que durante la transición política en Barcelona se produjo el primer boom atlético. Otro eje explicativo de su éxito fue el carácter de estas carreras. Más allá de limitarse a ser una expresión deportiva, incorporaron algunos elementos como el anhelo de libertad, de democracia y de reivindicación, todos ellos característicos de la transición política. Ello también evidenció algunas diferencias entre las carreras que mantenían un formato más competitivo - como la Maratón de Barcelona o la Jean Bouin- y las carreras surgidas durante la transición. Desde el punto de vista reivindicativo, fueron las carreras organizadas por las asociaciones de vecinos las que se dotaron de mayor intencionalidad en este sentido y del descontento ciudadano hacia determinadas demandas sociales. Un último eje característico de las carreras populares es el correspondiente a los retos organizativos a los que se hizo frente como consecuencia de su éxito y de la inexperiencia organizativa. La presencia de numerosos errores y la introducción de cambios organizativos fue una constante de la transición política, algo que condujo a la necesidad de priorizar la formación y la publicación de textos que facilitaban orientaciones organizativas de eventos deportivos al aire libre.

Como línea de investigación futura, se propone completar este análisis mediante la realización de entrevistas en profundidad a los principales responsables políticos y organizadores de las carreras populares surgidas durante la transición política.

\section{Referencias}

Abadia, S. (2011 a). Deporte, ciudadanía y libertad: la transición política en España y el deporte (1975-1982). En X. Pujadas (Coord.), Atletas y ciudadanos. Historia social del deporte en España: 1870-2010 (pp. 357-392). Madrid: Alianza Editorial.

Abadia, S. (2011 b). El moviment veïnal i la promoció de l'esport a la Barcelona de la Transició Democràtica (1975-1982). Barcelona: Consell Català de I'Esport. Generalitat de Catalunya.

Alfil (1977, 17 de diciembre). Con el pleno termina la I Asamblea del Deporte. El Mundo Deportivo, p. 20.

Ajuntament de Barcelona (1983). Macroàrea de Serveis Personals. Memòria d'activitats 1982. Barcelona: Ajuntament de Barcelona.

Associació de Veïns de Sant Andreu (1977, diciembre). Programa popular "Festa Major 1977". En Sant Andreu. Butlletí de l'Associació de Veïns.

Associació de Veïns de Sarrià (1978, octubre). Festes del Roser. Programa. En Sarrià. Circular interna de l'Associació de Veïns.

Associació de Veïns del Parc (1980, Junio). Esports. En Butlletí de I'Associació de Veïns del Parc. 
Abadia, S. (2014). Las carreras populares en la Barcelona de la transición política (1975-1982). RICYDE.

Astruells, A. (1979, 24 de diciembre). Barcelona: ¿es una ciudad deportiva? El Mundo Deportivo, p. 25.

Ateneu Enciclopèdic "Sempre Avant" (1979, octubre). Cultura Física. En Butlletí Informatiu.

Barcelona despedirá el año corriendo (1978, 28 de diciembre). El Mundo Deportivo, p. 24.

Barcelona se pone a correr con la I carrera popular (1979, 25 de maig). El Mundo Deportivo, p. 31.

Bernecker, W. L. (2009). El cambio de mentalidad en el segundo franquismo. En N. Townson (Ed.), España en cambio. El segundo franquismo, 1959-1975 (pp. 49-70). Madrid: Siglo XXI de España Editores.

C. M. (1981, 28 de abril). Una carrera para la ciudad. Dicen..., p. 41.

Cánovas venció en la "Cursa" de "El Corte Inglés" (1982, 17 de mayo). El Mundo Deportivo, p. 36.

Casasa, J. M. y Durà, J. (1984). La promoció de l'Esport comença a l'escola. Barcelona.

Carbonell, F. (1981, 25 de mayo). La "bici", protagonista en Barcelona. Dicen..., p. 47.

Carreras populares en el distrito Quinto (1978, 30 de septiembre). El Mundo Deportivo, p. 24.

Cursos del CSD en Barcelona (1978, 26 de noviembre). El Mundo Deportivo, p. 30.

Dalmases, J. (1977, 14 de diciembre). La bicicleta en lucha: las bicicletas se levantan en favor de las bicicletas. El Mundo Deportivo, p. 22.

Declaración programática de la D.N.D. (1976, 3 de octubre). El Mundo Deportivo, p. 3.

Duran, S. (1982, 6 de diciembre). Los VIP'S, satisfechos. El Mundo Deportivo, p. 39.

E. M. (1980, 15 de febrero). "Footing": Deporte en la calle. Dicen..., p. 25.

En marcha, la II carrera popular "Ciutat de Barcelona" (1980, 4 de mayo). El Mundo Deportivo, p. 38.

F. C. (1981, 9 de mayo). 30.000 corredores en la $3^{a}$ carrera popular "Ciutat de Barcelona". El Mundo Deportivo, p. 31.

F. C. (1982, 24 de septiembre). Barcelona se pone en marcha. El Mundo Deportivo, p. 26.

Fotut pel footing (1980, mayo-junio). En Hortavui, Portaveu del Barri.

García, M. (2001). Los españoles y el deporte: prácticas y comportamientos en la última década del siglo XX. Encuesta sobre hábitos deportivos de los españoles 2000. Madrid: Consejo Superior de Deportes.

García, M. (2006). Veinticinco años de análisis del comportamiento deportivo de la población española (1980-2005). Revista Internacional de Sociología, 44, 15-38.

Gaseta Municipal de Barcelona (1979). Número 1457.

Glover, B. y Shepherd, J. (1979). Correr para vivir mejor. Barcelona: Círculo de Lectores.

Hempel, T. (1981). La práctica del jogging. Barcelona: Formentera.

Hewitt, J. (1980). Jogging. Barcelona: Bruguera.

Huertas, J. M. y Andreu, M. (1996). Barcelona en lluita: el moviment urbà 1965-1996. Barcelona: Federació d'Associacions de Veïns de Barcelona. 
Abadia, S. (2014). Las carreras populares en la Barcelona de la transición política (1975-1982). RICYDE.

Huntington, S. P. (1994). La tercera ola. La democratización a finales del siglo XX. Barcelona: Paidós.

ICSA-Gallup (1975). El Español y el Deporte: datos de una encuesta. Madrid: Delegación Nacional de EF y Deportes.

Instalaciones deportivas en todos los pueblos españoles objetivo de la DND (1976, 7 de enero). Dicen..., p. 16.

Jean Bouin: La Carrera Popular, novedad de este año (1979, 16 de noviembre). El Mundo Deportivo, p. 30.

La democratización a encuesta (1976, 7 de octubre). Dicen..., p. 8.

La gran fiesta atlética (1980, 7 de diciembre). El Mundo Deportivo, p. 38.

La IV "Cursa" de la Merced, record de participación (1982, 23 de septiembre). El Mundo Deportivo, p. 24.

Marcos, J. F. (1981). Deporte para todos. Madrid: Diputación Provincial de Madrid.

Martín, L. (Ed.). (1996). Constitución Española. Pamplona: Aranzadi.

Messeguer se impuso en la "Marathon" (1980, 1 de octubre). El Mundo Deportivo, p. 36.

Moscoso, D. (2011). Los españoles y el deporte. Del pódium al banquillo. Panorama Social, 14, 110-126.

Muntaner, E. (1978, 4 de diciembre). 54 edición "Jean Bouin" iBravo! Cerrada. Dicen..., p. 35.

Muntaner, E. (1980, 8 de diciembre). England, England!! Dicen..., p. 32.

Muntaner, E. (1981, 21 de enero). La seriedad debe imperar en la "Barcelona-81". Dicen..., p. 14.

Nash, M. (2002). Identitats, espais socials i multiculturalisme: visions del passat i del present. En C. Fauria y Y. Aixelà (Coords.), Barcelona, mosaic de cultures (pp. 3548). Barcelona: Museu Etnològic de Barcelona.

Presentación oficial de la "IV Cursa Atlética de la Mercè" (1982, 18 de septiembre). El Mundo Deportivo, p. 31.

Puig, N. (1981). L'espai esportiu català. En M. Campo Vidal (Coord.), L'esport de base a Catalunya (pp. 45-66). Barcelona: Ketres.

Puig, N. (1993). Revisión histórica de la política deportiva en España, lecciones que se pueden extraer de cara al futuro. En $3^{\circ}$ Encuentros de política deportiva (pp. 93105). Barakaldo: Instituto Municipal de Deportes. Ayuntamiento de Barakado.

Puig, N. (1995). Esport i societat a Catalunya. En L'Associacionisme i l'esport (pp. 251264). Barcelona: Secretaria General de I'Esport.

Puig, N. y Heinemann, K. (1995). Institucions públiques i desenvolupament de l'esport a Catalunya (1975-1994). Proposta d'un marc teòric interpretatiu. Acàcia, 4, 123-143.

Pujadas, X. y Santacana, C. (1999). Esport, espai i societat en la formació urbana de Barcelona (1870-1992). Barcelona: Fundació Barcelona Olímpica.

Pujol, P. (Coord.). (1981). Del "jogging" a la maratón. Barcelona: Espaxs.

Santacana, C. (2003). Una interpretació de l'esport a Catalunya. Lluc, 835-836, 35.

Segona Cursa de la Mercè. Toda Barcelona se puso a correr (1980, 25 de septiembre). El Mundo Deportivo, p. 30.

Segura, F. (1981). L'administració no entén l'esport base. En M. Campo Vidal (Coord.), L'esport de base a Catalunya (pp. 32-44). Barcelona: Ketres. 
Abadia, S. (2014). Las carreras populares en la Barcelona de la transición política (1975-1982). RICYDE.

Soto, A. (2005). Transición y cambio en España. 1975-1996. Madrid: Alianza Editorial.

Vilanou, C. (1994). L'esport a Sants-Montjuïc. Una visió histórica (1883-1994). Barcelona: Arxiu Municipal del Districte de Sants-Montjuïc.

Vilaseca: "Un encuentro con la tradición" (1980, 8 de diciembre). El Mundo Deportivo, p. 48.

25.000 Barceloneses, en la "Cursa de la Mercè" (1979, 23 de septiembre). El Mundo Deportivo, p. 27.

40 minusválidos correrán en silla de ruedas (1978, 18 de noviembre). El Mundo Deportivo, p. 27. 